\title{
Alcohol use disorder and tuberculosis treatment: A longitudinal mixed method study in Thailand
}

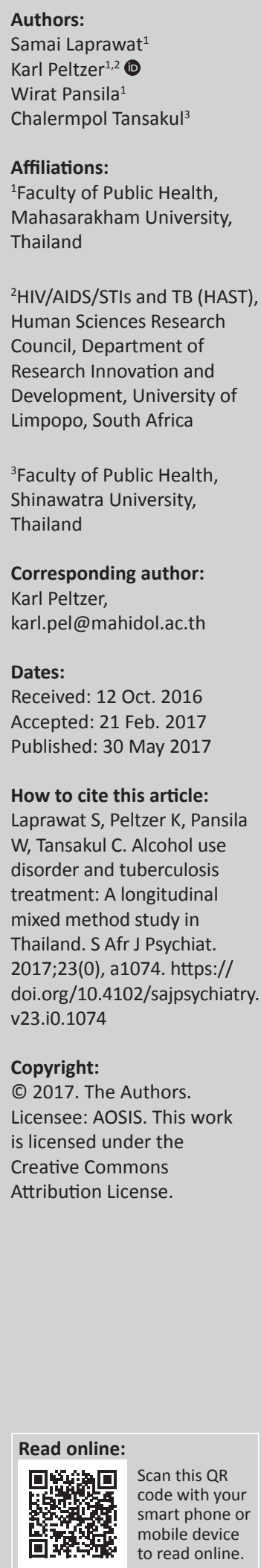

Objective: The relationship between tuberculosis (TB) treatment and alcohol use disorders over time is under-researched. The aim of this investigation was to study alcohol use and TB medication adherence and its predictors among TB patients over a period of 6 months.

Methods: A longitudinal investigation was carried out with new TB and TB retreatment patients systematically selected from two hospitals and had screened positive for hazardous or harmful alcohol use in Sisaket Province in Thailand. Alcohol use disorders were measured with Alcohol Use Disorder Identification Test (AUDIT)-C at baseline, 3 months and 6 months.

Results: Of the 295 TB patients who were screened with AUDIT-C, 72 (24.4\%) tested positive for hazardous or harmful alcohol use. At 6 months, 72 TB patients had completed the followup. At the 6-month follow-up, hazardous or harmful drinking was reduced by $84.7 \%$. Multivariate logistic regression analysis using generalised estimation equation modelling found that alcohol use significantly reduced over time, whereas there was no change in current tobacco use.

Conclusion: The prevalence of alcohol use disorders significantly reduced over a period of 6 months.

\section{Introduction}

Thailand is a high-burden tuberculosis (TB) country; the TB case detection rate was $80.0 \%$ and the TB treatment success rate was $81.0 \% .^{1}$ In Thailand, the age-standardised prevalence of hazardous or harmful alcohol use was $16.6 \%$ among men and $2.1 \%$ among women, and current smokers were $45.8 \%$ among men and $2.3 \%$ among women. ${ }^{2}$ In a study among male hospital outpatients in central Thailand, the proportion of moderate or high-risk alcohol users was $44.2 \%$, and the proportion of moderate or high-risk tobacco users was $55.8 \%{ }^{3}$

In cross-sectional studies, high prevalences of alcohol use disorders have been found among TB patients. ${ }^{4,5}$ Alcohol use has been found associated with TB treatment default, ${ }^{6,7,8,9}$ multidrugresistant $\mathrm{TB}^{10}$ and the hazard for an unsuccessful outcome of multidrug-resistant $\mathrm{TB} \cdot{ }^{11,12}$ It is unclear whether alcohol disorders reduce, stay the same or increase after initiating TB treatment. There is a lack of longitudinal studies on alcohol use disorders in TB patients during their TB treatment, and the nature of comorbidity between alcohol use and other mental disorders such as depression and smoking during TB treatment is not well understood..$^{13}$ For example, in a brief alcohol intervention trial among TB patients in South Africa, alcohol use reduced significantly from the beginning of TB treatment to 6-month follow-up in the control group. ${ }^{14}$

In relation to other mental disorders, including major depressive disorder, which often co-exist with alcohol use disorders, several studies have found an improvement of mental health scores during the course of TB treatment. ${ }^{15,16,17,18,19,20,21,22}$ Some of the researchers ${ }^{19,20,21,22}$ explain this decline in mental symptoms with the general physical improvement during TB treatment that subsequently improves mental well-being. Regarding tobacco use disorders, another comorbidity of alcohol use disorders, two smoking intervention studies in Nepal and Pakistan found no reduction of smoking in the control group. ${ }^{23,24}$ The results from these studies seem to support the idea that routine interventions by health care providers do not prevent tobacco use among TB patients. However, in a large alcohol intervention trial with TB patients in South Africa, daily or almost daily tobacco use significantly reduced over time during TB treatment. ${ }^{25}$

The relationship between TB and alcohol use during the time of TB treatment needs more research. Therefore, the aim of this investigation was to estimate the prevalence of alcohol use disorder severity and its predictors among TB patients over a period of 6 months in Thailand. 


\section{Methods Study design}

This is a longitudinal, mixed observational study of TB patients with hazardous or harmful drinking problems in two public district hospitals in Sisaket Province in northeastern Thailand.

\section{Sample and procedure}

Adult new TB and new retreatment patients with hazardous or harmful alcohol use from two hospitals in Sisaket Province in Thailand were consecutively interviewed after informed consent was obtained within 1 month of starting anti-TB treatment and were followed up at 6 months of treatment in 2015-2016. There were no exclusion criteria. Quantitative information was collected for 72 patients, and qualitative information was collected for a sub-sample of 20 patients. Every third TB patient recruited for the quantitative interview was included for the qualitative interview until a sample size of 20 was reached. Quantitative and qualitative individual interviews were conducted in Thai language. The measures for alcohol use and depression were available in Thai language, and the remaining sections were translated from English to Thai and back-translated by two independent bilingual translators.

\section{Measures \\ Quantitative}

Hazardous or harmful alcohol use was assessed with the Alcohol Use Disorder Identification Test (AUDIT)-C, using a cut-off score of 3 for women and 4 for men. ${ }^{26}$ The Thai version of the AUDIT has been validated in Thailand. ${ }^{27}$ The Cronbach alpha for the AUDIT-C in this study was 0.89 at Time 1, 0.97 at Time 2 and 0.92 at Time 3.

Tobacco use was assessed with two questions: (1) 'Do you currently smoke any tobacco products, such as cigarettes, cigars or pipes?' and (2) 'Do you currently use any smokeless tobacco, such as snuff, chewing tobacco, betel?' Response options were 'yes' or 'no'..$^{28}$

Depression was assessed with a Thai-validated nine-item Primary Health Questionnaire (PHQ-9) (Thai version - local language). ${ }^{29}$ A score of 10 and more was used to classify moderate-to-severe depressive symptoms. The Cronbach alpha for the PHQ-9 in this study was 0.70 at Time 1, 0.82 at Time 2 and 0.72 at Time 3.

Anti-TB medication adherence was assessed with the question, 'In the past 10 days, on how many days did you not take your TB medication?' Taking less than 90\% anti-TB medication was classified as non-adherence to TB medication. ${ }^{30}$

Health variables such as TB treatment status and HIV status were assessed by interviews and from medical file information.
Socioeconomic characteristics included age, gender, educational level and monthly family income.

TB treatment outcomes were defined as successful treatment (defined as cured or completed treatment) or unsuccessful treatment. ${ }^{31}$ The TB treatment protocol in Thailand follows WHO guidelines, including directly observed treatment (DOT) support, and provides health education on substance use. ${ }^{31}$

\section{Qualitative}

Guiding questions were adopted from Thomas et al. ${ }^{32}$ as follows:

In your opinion does alcohol use affect TB treatment compliance? What do you perceive as the problems you encounter because of alcohol use? Could you elaborate on reasons why you might have missed your TB medication because of alcohol use? (p. 3)

\section{Data analysis}

Data were analysed using the IBM Statistical Package for the Social Sciences (SPSS) for Windows version 23.0 (SPSS, Inc., Chicago, IL, USA). Frequencies, means and standard deviations were computed to describe the sample. Chi-square and Friedman tests were performed to compare repeated measures of alcohol use and depressive disorders at baseline and at 6-month follow-up. Multivariate logistic regression analysis was conducted using generalised estimation equation (GEE) modelling. Probability $<0.05$ was regarded as statistically significant. Qualitative data were analysed using thematic content analysis.

\section{Results}

\section{Sample characteristics}

Of the 295 TB patients who were screened with AUDIT-C, 72 (24.4\%) were found to be positive for hazardous or harmful alcohol use. At baseline, 72 TB patients with hazardous or harmful drinking and, at 6-month follow-up, 72 TB patients were included. Most of the participants were men $(90.3 \%)$, $9.7 \%$ were women, $75.0 \%$ were 41 years and older, $77.8 \%$ were married, $84.7 \%$ had a primary or less education and $34.7 \%$ had a monthly family income of 8000 or more Thai Baht. Regarding health variables, $97.2 \%$ of the sample were new TB patients, $2.8 \%$ were retreatment TB patients and $6.9 \%$ were HIV positive (Table 1).

\section{Alcohol use, tobacco use, depression and tuberculosis medication non-adherence at baseline, 3-month and 6-month follow-up}

At baseline, the mean AUDIT-C score was 8.6, which significantly reduced to 1.3 at 3 months and 1.0 at 6 months. The proportion of hazardous or harmful alcohol users also reduced significantly over time from $100.0 \%$ to $15.3 \%$. Similarly, past month binge drinking reduced from $88.9 \%$ at baseline to $13.9 \%$ at 6-month follow-up. Almost one in five $(18.1 \%)$ were current tobacco users at baseline, which remained almost the same over time $(24.1 \%$ at 3 months and 
$15.5 \%$ at 6 months). Moderate or severe depression significantly reduced from $25.0 \%$ at baseline to $11.1 \%$ at 6-month follow-up. TB medication non-adherence was $4.2 \%$ at baseline and 6-month follow-up. Of the 72 TB patients, 70 successfully completed the treatment and 2 defaulted (Table 2).

\section{Predictors of hazardous or harmful drinking and binge drinking}

In multivariate logistic regression analysis using GEE modelling, hazardous or harmful drinking and binge drinking significantly reduced over time. Further, current tobacco was positively associated with hazardous or harmful alcohol use and binge drinking. Due to too few cases of women, TB non-medication adherence, HIV status and TB treatment outcome (default vs. completed or cured); these variables were not included in the model (Table 3).

\section{Qualitative results}

The qualitative results can be classified into three groups: (1) drinkers who reduced drinking at the beginning of $\mathrm{TB}$

\begin{tabular}{lcc} 
TABLE 1: Sample characteristics at 6 months of follow-up $(N=72)$ & \\
\hline Socio-demographics & $\boldsymbol{N}$ & $\%$ \\
\hline Gender & 65 & 90.3 \\
Male & 7 & 9.7 \\
Female & & \\
Age & 18 & 25.0 \\
18-40 & 30 & 41.7 \\
41-54 & 24 & 33.3 \\
55 or more & & \\
Marital status & 56 & 77.8 \\
Married & 16 & 22.2 \\
Single, widowed, separated, divorced & & \\
Education & 61 & 84.7 \\
Primary or less & 11 & 15.3 \\
Post primary & & \\
Family income (monthly in Thai Baht) $\dagger$ & 18 & 25.0 \\
0-4999 & 29 & 40.3 \\
5000-7999 & 25 & 34.7 \\
8000 or more & & \\
Health variables & 70 & 97.2 \\
New TB patient & 2 & 93.1 \\
Retreatment TB patient & 57 & \\
HIV negative & & \\
HIV positive/coinfected & & \\
\hline
\end{tabular}

\section{TB, tuberculosis.}

$\dagger$, One US\$ $=36$ Thai Baht. treatment but increased after improving, (2) drinkers who eventually stopped drinking and (3) drinkers who continued drinking:

(1) In a number of patients, there was a tendency to reduce or even stop drinking alcohol at the beginning of TB treatment, , but after 3 or 4 months of treatment, when their general health improved, they resorted to increased use of alcohol:

'Sometimes I accidentally drank a lot and forgot taking the pills.' (Man, 39 years old)

'After work I used to drink with colleagues, but not so frequently. When I felt sick, I used I quit drinking. After my body [physical well-being] had improved, I started drinking with my friends again.' (Man, 35 years old)

(2) Another group of patients stopped drinking and their relationship with the family and community improved:

'I stopped drinking since I got TB. The doctor recommended not drinking alcohol in order to recover. I am aware that alcohol can cause side effects of the drugs harming my body.' (Man, 49 years old)

TABLE 3: Predictors of hazardous or harmful drinking and binge drinking.

\begin{tabular}{|c|c|c|}
\hline \multirow[t]{2}{*}{ Variables } & $\begin{array}{c}\text { Hazardous or } \\
\text { harmful drinking }\end{array}$ & $\begin{array}{c}\text { Binge } \\
\text { drinking }\end{array}$ \\
\hline & $\operatorname{AOR}(95 \% \mathrm{Cl}) \dagger$ & AOR $(95 \% \mathrm{Cl})$ \\
\hline \multicolumn{3}{|l|}{ Socio-demographics } \\
\hline \multicolumn{3}{|l|}{ Age in years } \\
\hline $18-40$ & 1 (Reference) & 1 (Reference) \\
\hline $41-54$ & $0.93(0.86-1.01)$ & $0.93(0.81-1.06)$ \\
\hline 55 or more & $1.00(0.93-1.08)$ & $1.05(0.91-1.21)$ \\
\hline \multicolumn{3}{|l|}{ Marital status } \\
\hline Single, widowed, separated, divorced & 1 (Reference) & 1 (Reference) \\
\hline Married & $1.03(0.93-1.13)$ & $1.02(0.87-1.18)$ \\
\hline \multicolumn{3}{|l|}{ Education } \\
\hline Primary or less & 1 (Reference) & 1 (Reference) \\
\hline Post primary & $0.96(0.86-1.07)$ & $0.93(0.78-1.09)$ \\
\hline \multicolumn{3}{|l|}{ Current tobacco use } \\
\hline No & 1 (Reference) & 1 (Reference) \\
\hline Yes & $1.16(1.02-1.33)^{*}$ & $1.24(1.09-1.42)^{* * *}$ \\
\hline Depression (moderate to severe) & $1.16(1.02-1.33)$ & $0.97(0.81-1.15)$ \\
\hline \multicolumn{3}{|l|}{ Time } \\
\hline Baseline ( $\leq 1$ month on TB treatment) & 1 (Reference) & 1 (Reference) \\
\hline Time 2 (6 months) & $0.43(0.40-0.47)^{* * *}$ & $0.47(0.43-0.53)^{* * *}$ \\
\hline
\end{tabular}

$* * * p<0.001 ; * p<0.05$

AOR, adjusted odds ratio; $\mathrm{Cl}$, confidence interval.

$\dagger$, Goodness of fit: quasi-likelihood under independence model criterion (QIC): 23.21.

$\$$, Goodness of fit: QIC: 31.18 .

TABLE 2: Change in alcohol, tobacco use, depression and TB medication non-adherence over 6-month follow-up ( $N=72$ ).

\begin{tabular}{|c|c|c|c|c|c|c|c|c|}
\hline \multirow[t]{2}{*}{ Variables } & \multicolumn{2}{|c|}{$\begin{array}{l}\text { At baseline } \\
(N=72)\end{array}$} & \multicolumn{2}{|c|}{$\begin{array}{c}3 \text { months } \\
(N=30)\end{array}$} & \multicolumn{2}{|c|}{$\begin{array}{l}6 \text { months } \\
(N=72)\end{array}$} & \multirow{2}{*}{$\begin{array}{c}\text { Change from } \\
\text { baseline to } \\
6 \text { months }(M / \%)\end{array}$} & \multirow[t]{2}{*}{$p$} \\
\hline & $M(\mathrm{SD})$ & $N(\%)$ & $M(\mathrm{SD})$ & $N(\%)$ & $M(\mathrm{SD})$ & $N(\%)$ & & \\
\hline AUDIT-C score & $8.6(2.5)$ & & $1.3(3.6)$ & & $1.0(2.6)$ & & -7.6 & $<0.001$ \\
\hline Hazardous or harmful alcohol use & & $72(100)$ & & $6(20.0)$ & & $11(15.3)$ & -84.7 & $<0.001$ \\
\hline Binge drinking (past month) & & $64(88.9)$ & & $6(20.0)$ & & $10(13.9)$ & -75.0 & $<0.001$ \\
\hline Current tobacco use & & $13(18.1)$ & & $7(24.1)$ & & $11(15.5)$ & -2.6 & ns \\
\hline Moderate or severe depression (PHQ-9 $=\geq 10$ ) & & $18(25.0)$ & & $11(36.7)$ & & $8(11.1)$ & -13.9 & $<0.001$ \\
\hline TB medication non-adherence & & $3(4.2)$ & & $2(6.7)$ & & $3(4.2)$ & 0.0 & ns \\
\hline Treatment outcome & \multicolumn{8}{|c|}{70 completed or cured 2 defaulted } \\
\hline
\end{tabular}

AUDIT-C, Alcohol Use Disorder Identification Test; PHQ-9, nine-item Primary Health Questionnaire; TB, tuberculosis. 
'I used to drink before going to the clinic. The health officers smelled alcohol and complained that I had to stop drinking. I explained that I would try, but then later I stopped drinking.' (Man, 38 years old)

(3) A third group of patients continued drinking alcohol:

'I continued drinking until the next morning. This gives me trouble and affects my drug taking.'

'Alcohol affects you. I do not think about it. I have a drinking problem; I live apart from my wife. Some days I forget to take my medicine.'

\section{Ethical consideration}

The study protocol was approved by the Faculty of Public Health, Mahasarakham University Ethics Committee (No. PH 003/2559) and the Ministry of Public Health of Sisaket Province, Thailand.

\section{Discussion}

This prospective cohort study found a significant decline in the prevalence of alcohol use disorder among TB patients over a 6-month period, as also found in the control group of a brief alcohol intervention trial among TB patients in South Africa. ${ }^{14}$ Possible explanations for the decline of alcohol use in this study may be attributed to (1) the intervention effect of standard care (health care worker's advice to reduce or stop drinking alcohol), (2) the negative experience of drinking and taking anti-TB medication, as found from the qualitative interviews in a sub-sample of the studied TB patients and (3) natural history changes in drinking over time in the course of TB treatment. ${ }^{33}$

Further, this study in agreement with several previous studies $15,16,17,18,19,20,21,22$ found that mental well-being improved or depressive symptoms reduced during the course of TB treatment.

The reduction of depressive symptoms could be explained by the improvement of the physical health status over the duration of treatment, which then could also improve the mental well-being. ${ }^{19,20,21,22}$

However, this study found that tobacco use remained unchanged over the course of TB treatment. This finding is in agreement with two previous studies ${ }^{23,24}$ and in disagreement with another study that found a decline of tobacco use during TB treatment, ${ }^{25}$ while, in a study in Indonesia, most TB patients were found to stop smoking when under TB treatment but a large group would relapse to smoking posttreatment. ${ }^{34}$ In multivariable analysis, tobacco use but not depression symptoms was associated with hazardous or harmful alcohol use and binge drinking in this study. A high degree of association between alcohol and nicotine dependence has been pointed out earlier. ${ }^{35,36}$ As both alcohol and tobacco use, in particular, negatively impact on TB treatment, dual interventions including alcohol and tobacco may be indicated.$^{36}$ Other authors ${ }^{37}$ suggest to better integrate TB and mental health services.

\section{Study limitations}

Generalisability of our findings is limited to TB patients with alcohol problems on anti-TB treatment in hospital facilities in Sisaket Province in Thailand. A further limitation was that most study variables were assessed by interviews, and socially desirable responses may have been provided.

\section{Conclusion}

The prevalence of alcohol use in this study among TB patients significantly reduced over a 6-month period.

\section{Acknowledgements Competing interests}

The authors declare that they have no financial or personal relationships which may have inappropriately influenced them in writing this article.

\section{Authors' contributions}

S.L., K.P., W.P. and C.T. designed the study. S.L. conducted data collection. S.L. and K.P. analysed the data and wrote the paper. All authors read and approved the final manuscript.

\section{References}

1. World Health Organization. Global tuberculosis report 2014 [homepage on the Internet]. 2014 [cited 2016 Dec 10]. Available from: http://apps.who.int/iris/ bitstream/10665/137094/1/9789241564809_eng.pdf

2. Aekplakorn W, Hogan MC, Tiptaradol S, Wibulpolprasert S, Punyaratabandhu $P$, Lim SS. Tobacco and hazardous or harmful alcohol use in Thailand: Joint prevalence and associations with socioeconomic factors. Addict Behav. 2008;33:503-514. https://doi.org/10.1016/j.addbeh.2007.10.010

3. Pengpid S, Peltzer K, Puckpinyo A, Thammaaphiphol K. Conjoint moderate or high risk alcohol and tobacco use among male out-patients in Thailand. S Afr J Psychiatry. 2016;22(1):a763. https://doi.org/10.4102/sajpsychiatry.v22i1.763

4. Peltzer K, Louw J, McHunu G, et al. Hazardous and harmful alcohol use and associated factors in tuberculosis public primary care patients in South Africa. Int J Environ Res Public Health. 2012;9:3245-3257. https://doi.org/10.3390/ ijerph9093245

5. Suhadev M, Thomas BE, Raja Sakthivel M, et al. Alcohol use disorders (AUD) among tuberculosis patients: A study from Chennai, South India. PLoS One. 2011;6(5):e19485. https://doi.org/10.1371/journal.pone.0019485

6. Lackey B, Seas C, Van der Stuyft P, et al. Patient characteristics associated with tuberculosis treatment default: A cohort study in a high-incidence area of Lima, Peru. PLoS One. 2015;10(6):e0128541. https://doi.org/10.1371/journal. pone.0128541

7. Muture BN, Keraka MN, Kimuu PK, et al. Factors associated with default from treatment among tuberculosis patients in Nairobi province, Kenya: A case control study. BMC Public Health. 2011;11:696. https://doi.org/10.1186/14712458-11-696

8. Sendagire I, Schim Van der Loeff M, Kambugu A, et al. Urban movement and alcohol intake strongly predict defaulting from tuberculosis treatment: An operational study. PLoS One. 2012;7(5):e35908. https://doi.org/10.1371/journal. pone.0035908

9. Slama K, Tachfouti N, Obtel M, et al. Factors associated with treatment default by tuberculosis patients in Fez, Morocco. East Mediterr Health J. 2013;19: 687-693.

10. Jitmuang $A$, Munjit $P$, Foongladda $S$. Prevalence and factors associated with multidrug-resistant tuberculosis at Siriraj Hospital, Bangkok, Thailand. Southeast Asian J Trop Med Public Health. 2015;46:697-706.

11. Duraisamy K, Mrithyunjayan S, Ghosh S, et al. Does alcohol consumption during multidrug-resistant tuberculosis treatment affect outcome? A population-based study in Kerala, India. Ann Am Thorac Soc. 2014;11:712-718. https://doi. org/10.1513/AnnalsATS.201312-4470C

12. Kendall EA, Theron D, Franke MF, et al. Alcohol, hospital discharge, and socioeconomic risk factors for default from multidrug resistant tuberculosis treatment in rural South Africa: A retrospective cohort study. PLoS One. 2013;8:e83480. https://doi.org/10.1371/journal.pone.0083480

13. Ambaw F, Mayston R, Hanlon C, et al. Depression among patients with tuberculosis: Determinants, course and impact on pathways to care and treatment outcomes in a primary care setting in southern Ethiopia - A study protocol. BMJ Open. 2015;5:e007653. https://doi.org/10.1136/bmjopen-2015-007653 
14. Peltzer K, Naidoo P, Louw J, et al. Screening and brief interventions for hazardous and harmful alcohol use among patients with active tuberculosis attending primary public care clinics in South Africa: Results from a cluster randomized primary public care clinics in South Africa: Results from a cluster randomized
controlled trial. BMC Public Health. 2013:13:699. https://doi.org/10.1186/1471 2458-13-699

15. Jaber AA, Khan AH, Syed Sulaiman SA, Ahmad N, Anaam MS. Evaluation of health-related quality of life among tuberculosis patients in two cities in Yemen. PLoS One. 2016:11(6):e0156258. https://doi.org/10.1371/journal. pone.0156258

16. Das $M$, Isaakidis $P$, Van den Bergh R, et al. HIV, multidrug-resistant TB and depressive symptoms: When three conditions collide. Glob Health Action. 2014;7:24912. https://doi.org/10.3402/gha.v7.24912

17. Atif $M$, Sulaiman SA, Shafie AA, et al. Impact of tuberculosis treatment on health-related quality of life of pulmonary tuberculosis patients: A follow-up study. Health Qual Life Outcomes. 2014;12:19. https://doi.org/10.1186/14777525-12-19

18. Ugarte-Gil C, Ruiz P, Zamudio C, et al. Association of major depressive episode with negative outcomes of tuberculosis treatment. PLoS One. 2013;8(7):e69514. https://doi.org/10.1371/journal.pone.0069514

19. Vega P, Sweetland A, Acha J, et al. Psychiatric issues in the management of patients with multidrug-resistant tuberculosis. Int J Tuberc Lung Dis. 2004 8(6):749-759.

20. Louw JS, Mabaso M, Peltzer K. Change in health-related quality of life among pulmonary tuberculosis patients at primary health care settings in South Africa: A prospective cohort study. PLoS One. 2016;11(5):e0151892. https://doi. org/10.1371/journal.pone.0151892.eCollection 2016

21. Peltzer K. Decline of common mental disorders over time in public primary care tuberculosis patients in South Africa. Int J Psychiatry Med. 2016;51:236-245.

22. Deribew A, Deribe K, Reda AA, Tesfaye M, Hailmichael Y, Maja T. Do common mental disorders decline over time in TB/HIV co-infected and HIV patients without TB who are on antiretroviral treatment. BMC Psychiatry. 2013;13:174. https://doi. org/10.1186/1471-244X-13-174

23. Campbell IA, Chaudhary RD, Holdsworth GM, Lyne OD. Brief advice to tuberculosis patients in Nepal to stop smoking: A pilot study by the Britain Nepal Medical Trust. Int J Tuberc Lung Dis. 2014;18(12):1438-1442. https://doi.org/10.5588/ijtld. 14.0358

24. Siddiqi K, Khan A, Ahmad $M$, et al. Action to stop smoking in suspected tuberculosis (ASSIST) in Pakistan: A cluster randomized, controlled trial. Ann Int Med. 2013;158(9):667-675. https://doi.org/10.7326/0003-4819-158-9-20130507000006
25. Peltzer K. Correlates of tobacco use among tuberculosis patients in South Africa: A brief report. J Psychol Afr. 2016;26(5):473-476. https://doi.org/10.1080/14330 237.2016.1219567

26. Bush K, Kivlahan DR, McDonell MB, et al. The AUDIT alcohol consumption questions (AUDIT-C): An effective brief screening test for problem drinking. Ambulatory Care Quality Improvement Project (ACOUIP). Arch Int Med. 1998;158:1789-1795. https://doi.org/10.1001/archinte.158.16.1789

27. Lapham SC, Brown P, Suriyawongpaisal P, Skipper BJ, Chadbunchachai W, Paisarnsilp S. Use of AUDIT for alcohol screening among emergency room patients in Thailand. Subst Use Misuse. 1999;34(13):1881-1895. https://doi.org/10 3109/10826089909039430

28. World Health Organization (WHO). Guidelines for controlling and monitoring the tobacco epidemic. Geneva, Switzerland: WHO; 1998.

29. Lotrakul M, Sumrithe S, Saipanish R. Reliability and validity of the Thai version of the PHQ-9. BMC Psychiatry. 2008;8:46. https://doi.org/10.1186/1471 244X-8-46

30. Awofeso N. Anti-tuberculosis medication side effects constitute major factor for poor adherence to tuberculosis treatment. Bull World Health Organ. 2008;86(3):B-D.

31. Ministry of Public Health. NTP: National tuberculosis control programme guidelines, Thailand, 2013. Nonthaburi: Ministry of Public Health; 2013.

32. Thomas B, Suhadev M, Mani J, et al. Feasibility of an alcohol intervention programme for TB patients with alcohol use disorder (AUD) - A qualitative study from Chennai, South India. PLoS One. 2011;6:e27752. https://doi.org/10.1371/ journal.pone.0027752

33. McCambridge J, Kypri K. Can simply answering research questions change behaviour? Systematic review and meta analyses of brief alcohol intervention trials. PLoS One. 2011;6(10):e23748. https://doi.org/10.1371/journal.pone.0023748

34. Ng N, Padmawati RS, Prabandari YS, et al. Smoking behavior among former tuberculosis patients in Indonesia: Intervention is needed. Int J Tuberc Lung Dis. 2008;12:567-572.

35. Diehl A, Scherbaum N. Nicotine dependence as comorbidity of alcohol dependence - Epidemiology, etiology and therapy. Fortschr Neurol Psychiatr. 2008;76(1):14-20. https://doi.org/10.1055/s-2007-980076

36. Peltzer K. Conjoint alcohol and tobacco use among tuberculosis patients in public primary healthcare in South Africa. S Afr J Psychiatry. 2014;20(1):21-26. https:// doi.org/10.7196/SAJP.482

37. Mason PH, Sweetland AC, Fox GJ, Halovic S, Nguyen TA, Marks GB. Tuberculosis and mental health in the Asia-Pacific. Australas Psychiatry. 2016;24(6):553-555. https://doi.org/10.1177/1039856216649770 